\title{
Growth hormone treatment without a needle using the Preci-Jet 50 transjector
}

\author{
P Bareille, M MacSwiney, A Albanese, C De Vile, R Stanhope
}

\begin{abstract}
A new delivery system (Preci-Jet 50) which administers growth hormone through the skin using high pressure and without a needle was evaluated. This device was inconvenient and painful compared with a pen injection system. The conclusion is that the Preci-Jet is not the panacea for solving the problem of compliance with subcutaneous growth hormone injections.

(Arch Dis Child 1997;76:65-67)
\end{abstract}

Keywords: growth hormone treatment; transcutaneous high pressure administration (jet); needle phobia.

The number of children treated with growth hormone has increased dramatically over the last 10 years. ${ }^{1}$ Unfortunately, growth hormone treatment must be given by daily subcutaneous injections often for several years, posing obvious problems of acceptance and compliance. ${ }^{2}$ Two methods are commonly used for administering growth hormone instead of the traditional syringe and needle: a pen injection ${ }^{3}$ system and an autoinjector. ${ }^{4}$ The latter have certainly improved acceptability of growth hormone treatment. However, these devices involve the use of a needle. Knowing that the mere sight of a needle may be sufficient to cause distress, neither the pen nor the autoinjector have solved the problem of compliance for some children. One potential method of avoiding the use of a needle is a transcutaneous high pressure device (jet). Thus we have used a Preci-Jet originally designed for insulin ${ }^{5}$ and later adapted for growth hormone administration. This system administers the peptide hormone directly through the skin using high pressure and without a needle. It has been shown that plasma levels and absorption kinetics obtained with the Preci-Jet transjector are similar to those attained by cutaneous injection using a needle (personal communication: Serono Laboratories data). These data are in agreement with those reported for insulin. ${ }^{6}$ It is therefore appropriate to consider using the Preci-Jet in patients who have difficulty accepting repeated injections.

In this study, we report the results of a randomised crossover trial of Preci-Jet 50 against a pen injection system performed in 28 patients. Furthermore, we have evaluated the longer term outcome of a further eight children who have used the Preci-Jet for up to two years.

\section{Patients and methods}

Twenty eight patients (11 girls, 17 boys) were randomly selected from the endocrine clinic at Great Ormond Street Hospital for Children. All patients were receiving growth hormone treatment, 19 through an autoinjector and nine with needles and syringes. None was using a pen injector system at that time but nine had used one previously. The exclusion criteria were bleeding disorders and diseases of collagen synthesis. Mean age was 10.1 (range 5.7-16.7) years. The growth hormone dose range was from 15 to $30 \mathrm{IU} / \mathrm{m}^{2}$ body surface area/week. The subjects were randomly subdivided into two groups, A and B. Sex distribution was similar in both groups but mean age differed, with values of 8.7 and 11.2 years in groups A and B respectively. The study design was for each group to have growth hormone treatment using either the pen (Kabi Pen manufactured by Pharmacia Upjohn) or the Preci-Jet for six weeks followed by the alternative device. At the end of each six week period the patients completed a questionnaire in the presence of a growth research nurse, rating both general convenience and pain on a scale of -5 to +5 (the highest score corresponding to the most favourable response). At the end of the study the patients continued growth hormone administration with the method of their choice.

Eight children (two of whom participated in the above trial) carried on using the Preci-Jet for several months and were assessed separately. Their mean age at the onset of treatment with the jet was 9.3 (range 4-12) years.

The Preci-Jet 50 transjector was manufactured by Advanced Medical Technologies, Charlottetown, Prince Edward Island C1E $1 \mathrm{BO}, \mathrm{Canada}^{5}$ and is shown in fig 1 .

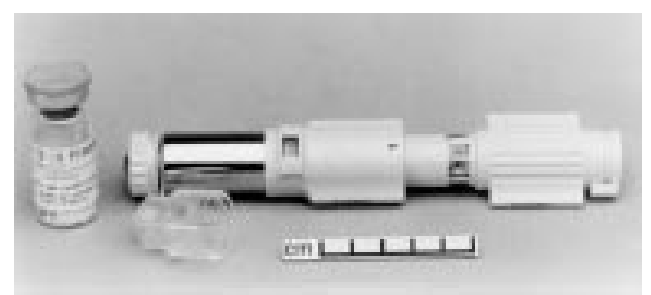

Figure 1 The Preci-fet 50 for transcutaneous administration of growth hormone. The nozzle for delivery of growth hormone is on the left of the device. A growth hormone ampoule and attachment for filling the device is illustrated. A centimetre rule is shown. The device propels fluid at high pressure through a fine orifice, which pierces the epidermis and spreads within the subcutaneous tissues. There are six pressure adjustment settings in the device and the most suitable setting for skin thickness and site of transjection is found by trial and error for the individual patient. 

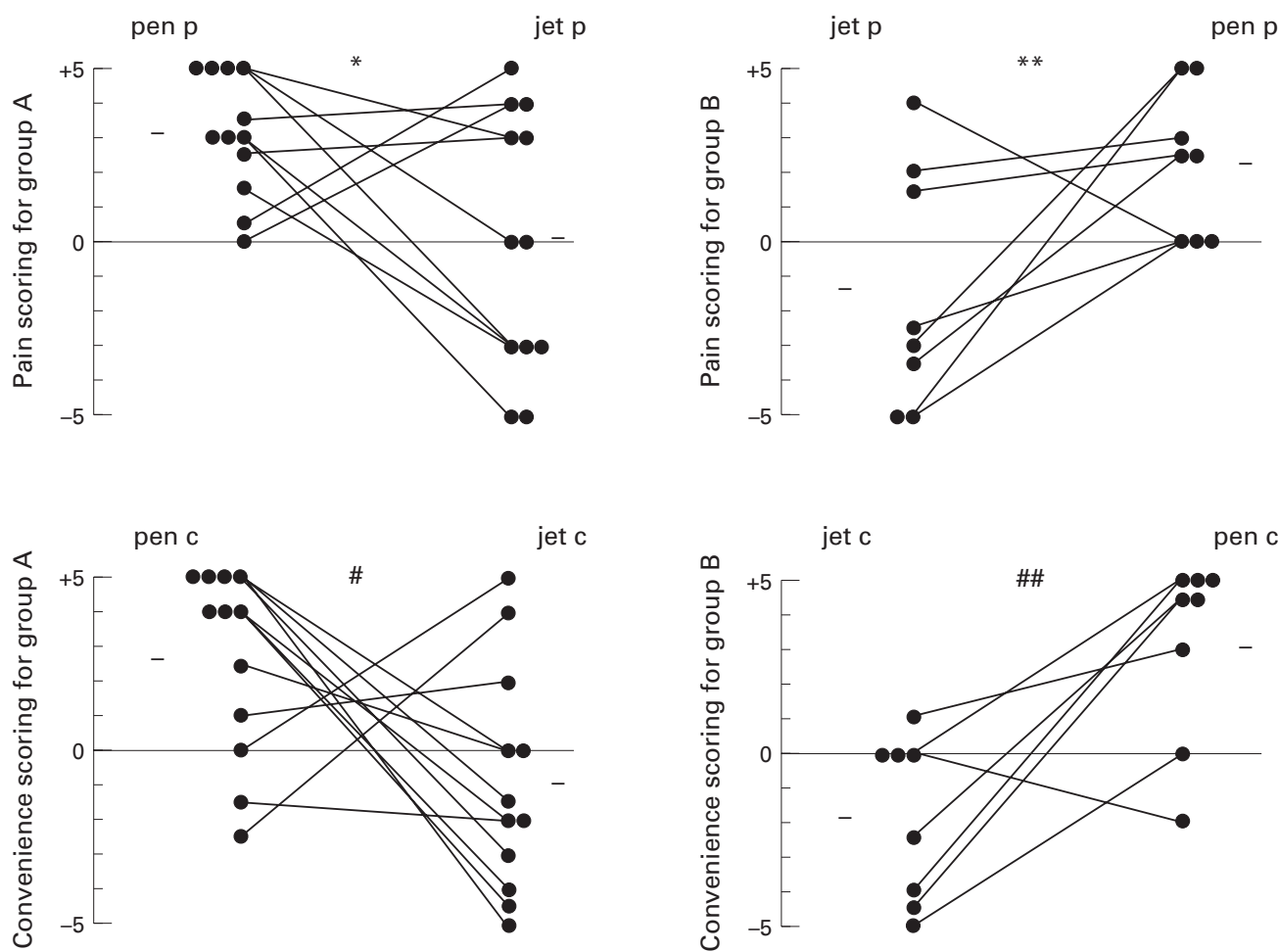

Figure 2 Individual results for pain scoring with the pen (pen $p$ ) or the jet (jet $p$ ) and for convenience scoring with the pen (pen c) or the jet (jet c) for groups $A(n=12)$ and $B(n=8) ;{ }^{*} p=0.04 ;{ }^{*} p=0.05 ; \# p=0.04 ; \# \# p=0.02$. In group $B$ one child completed the study but did not answer the questionnaire. Means are designated by bars.

Non-parametric methods (Wilcoxon matched pairs signed ranks test) were used for statistical analysis. Ethical committee approval was obtained at Great Ormond Street Hospital and a consent form signed by the parents/ guardians.

\section{Results}

At the end of the study, 12 patients continued with the pen whereas only two elected to use the Preci-Jet. Six returned to the autoinjector and one to needle and syringe. Of the seven children who did not complete the trial, five gave up the jet (excessive discomfort), one refused to surrender the jet, and another stopped growth hormone treatment.

The patients' scoring of the two methods of growth hormone administration is shown in fig 2 . The children found the pen less painful $(p \leqslant 0.05)$ and more convenient $(p \leqslant 0.04)$. The main reasons given for their decision to give up the jet were bruising and bleeding $(n=9)$ as well as time consuming and complicated to operate $(n=12)$. The absence of a needle was the main lure for those who decided to carry on with the jet.

In addition to the patients selected for the above trial, we have also used the Preci-Jet in eight patients with needle phobia for an average period of 8 (range 4-24) months. Only two children were still using the Preci-Jet at the time of inquiry and just for six months. All the others eventually abandoned it because of frequent bruises (predominant reason) and inconvenience. Two children had suffered from local skin blistering. One patient who had persisted with the Preci-Jet for two years was a survivor of malignant disease; his needle phobia had been the consequence of chemotherapy.

\section{Discussion}

This study shows a preference for the pen delivery system over the Preci-Jet, although this only achieved a low level of statistical significance. The rejection of the Preci-Jet is very clear when one considers that only three children of the 28 (one drop out and two who completed the trial) chose to continue with the Preci-Jet. The drawbacks of the Preci-Jet far outweighed the main potential advantage of the absence of a needle. Not only was it inconvenient, but it was also traumatic and frequently caused bleeding and pain. Five children could not tolerate using the jet and therefore withdrew from the trial. Nevertheless, a quarter of the patients appeared satisfied with the Preci-Jet (one drop out and six who completed the trial) but more than half of these eventually elected to use another mode of administration. The jet device could be, in certain patients, a temporary way of administering growth hormone before full acceptance of alternative methods. Children who persisted with the jet for one year or more were needle phobic as the result of previous chemotherapy for cancer or leukaemia. Despite this, they eventually returned to another mode of administration and overcame their fear of needles.

We conclude that the Preci-Jet 50, despite its absence of a needle, cannot replace the classical growth hormone delivery systems for the purpose of improving compliance. Nevertheless, this device may be beneficial to a selected group of needle phobic patients, 
particularly those who have received long term chemotherapy for treatment of malignant disease. Further development of transcutaneous jet devices, simpler to use and with more sensitive pressure settings, may render this method of administration more acceptable.

We are grateful to Serono (UK) for the loan of the Preci-Jet 50 transjectors. Drs Albanese, Bareille, and De Vile were supported by the Child Growth Foundation and M M by Pharmacia.

1 Drug and Therapeutics Commitee of the Lawson Wilkins Pediatric Endocrine Society. Guidelines for the use of growth hormone in children with short stature. $\mathcal{F}$ Pediatr 1995;127:857-67.
2 Smith SL, Hindmarsh PC, Brook CGD. Compliance with growth hormone treatment-are they getting it? Arch Dis Child 1993;68:91-3.

3 Gluckman PDG, Cutfield WS. Evaluation of a pen injector system for growth hormone treatment. Arch Dis Child 1991;66:686-8.

4 Stanhope R, Albanese A, Moyle L, Hamil G. Optimum method for administration of biosynthetic human growth hormone: a randomised crossover trial of an AutoInjector and a pen injection system. Arch Dis Child 1992;67:994-7.

5 Lindmayer I, Menassa K, Lambert J, et al. Development of new jet injector for insulin therapy. Diabetes Care 1986;9: 294-7.

6 Halle JP, Lambert J, Lindmayer I, et al. Twice-daily mixed regular and NPH insulin injections with new jet injector versus conventional syringes: pharmacokinetics of insulin absorption. Diabetes Care 1986;9:279-82. 